\title{
Characteristics of Musculoskeletal Involvement in Pediatric Patients with Disseminated Sepsis in a Tertiary Care Center
}

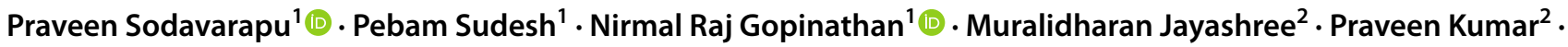 \\ Karthick Rangasamy ${ }^{1}$
}

Received: 6 April 2021 / Accepted: 13 August 2021 / Published online: 21 August 2021

(c) Indian Orthopaedics Association 2021

\begin{abstract}
Background Pediatric bone and joint infections account for one of the major causes of childhood morbidity. Disseminated sepsis being a systemic disorder with multisystem involvement, overshadows the timely recognition of bone and joint infections. Hence, we did this cross sectional study to evaluate the prevalence of septic arthritis and osteomyelitis in disseminated sepsis in children, the organisms implicated, and their antibiotic sensitivities.

Methods We prospectively collected data from 1st July 2016 to 31st September 2017 of children aged less than 12 years with disseminated sepsis, i.e., patients with fever and two or more sites of focal infection of anatomically non-contiguous tissues. Results Fifty-four patients of disseminated disease were included, of which 25 patients (46.3\%) had osteoarticular infections. Septic arthritis was seen in 17 patients, and osteomyelitis was seen in 12 patients. The most common joint was hip (41.6\%), and the most common bone involved was femur (53.8\%). Blood culture showed MRSA in 28\% and MSSA in 20\%. Joint and bone aspirates showed S. aureus in 56\% with $28 \%$ of MRSA and MSSA each. All Staphylococcus aureus organisms were found sensitive to vancomycin and teicoplanin. The mean values of CRP, duration of stay and duration of intravenous antibiotic was higher in MRSA infected patients compared to MSSA patients.

Conclusions Staphylococcus aureus is the most prevalent organism in musculoskeletal infection in disseminated sepsis children, with vancomycin sensitivity of $100 \%$ and methicillin sensitivity of $46.2 \%$ only. Cases of osteoarticular involvement with MRSA were higher compared to MSSA among the cases of disseminated disease. The prevalence of osteoarticular involvement is high in disseminated sepsis in children and increased clinical suspicion for such must be maintained.
\end{abstract}

Keywords Septic arthritis · Osteomyelitis · Disseminated infection $\cdot$ Staphylococcus aureus

Nirmal Raj Gopinathan

dr.nirmalraj78@gmail.com

Praveen Sodavarapu

praveen.omc.2k8@gmail.com

Pebam Sudesh

sudesh_lenica@rediffmail.com

Muralidharan Jayashree

mjshree@hotmail.com

Praveen Kumar

drpkumarpgi@gmail.com

Karthick Rangasamy

drsrk05@gmail.com

1 Department of Orthopaedics, Post Graduate Institute of Medical Education and Research, Sector 12, Chandigarh 160012, India

2 Department of Pediatrics, Post Graduate Institute of Medical Education and Research, Sector 12, Chandigarh 160012, India

\section{Introduction}

Pediatric bone and joint infection account for one of the major causes of childhood morbidity and mortality, with the incidence being up to 200 for osteomyelitis and 10-20 for septic arthritis per 1 lakh individuals in developing countries and mortality of up to $1 \%$ and morbidity up to $29 \%$ [1-3]. In children, these infections are most often caused by the dissemination of the pathogenic bacteria through blood [3]. Acute osteomyelitis can be a devastating disease unless it is diagnosed promptly and treated appropriately [3]. Septic arthritis causes morbidity due to the destruction of articular cartilage and underlying epiphysis with long term complications [4]. Timely diagnosis and appropriate treatment are cardinal to minimize complications and improve outcomes. The most common organism responsible for septic arthritis, acute osteomyelitis, and severe sepsis in children 
is Staphylococcus aureus [5, 6]. Sepsis is a systemic inflammatory response syndrome due to infection. Severe sepsis is defined as sepsis associated with organ dysfunction, hypoperfusion, or hypotension. Septic shock included hypoperfusion and hypotension despite adequate resuscitation [7]. Both acute osteomyelitis and septic arthritis can occur as skeletal manifestations in disseminated infection.

Disseminated sepsis being a systemic disorder with multisystem involvement, overshadows the timely recognition of bone and joint infection [7, 8]. Overall involvement and weakened immune system can preclude the classical presentation of musculoskeletal infection. This can lead to delay in diagnosis as well as persistent foci, with many times grave long term complications related to the limb. Early identification of these osteoarticular infections in disseminated sepsis with appropriate antibiotic therapy and surgical drainage is necessary to minimize morbidity. Since there is a lack of literature about the characteristics of such infections in disseminated sepsis, we did this cross-sectional study to evaluate the prevalence of septic arthritis and osteomyelitis in disseminated sepsis in children, the organisms implicated, and their antibiotic sensitivities.

\section{Materials and Methods}

After approval from the institute ethics committee (INT/ IEC/2017/000310), we prospectively collected data from Jul 1, 2016 to Sept 31, 2017, of all children of age less than 12 years presenting with disseminated infection. These are patients with fever and two or more sites of focal infection of anatomically non-contiguous tissues (skin, bone, joint, lung, heart, etc.) presenting to the Pediatric emergency of our tertiary care center in a developing country [7, 8]. Demographical details were collected and patients with clinical features suggestive of septic arthritis (fever, general malaise, painful passive joint motion) and acute osteomyelitis (fever, general malaise, local pain, and tenderness) during their entire stay in hospital were considered after appropriate clinical examination and were included using Morrey criteria and Peltola and Vahvanen criteria, respectively $[9,10]$. Erythrocyte sedimentation rate, C-reactive protein, total leukocyte count, differential leukocyte count, and blood culture were sent, and initial roentgenograms and an ultrasonographic examination were carried out. Joint and/or bone aspiration was done, and aspirated material were sent for gram stain, aerobic bacterial culture, TB culture and fungal culture and sensitivity. As per the institutional protocol, empirical antibiotic therapy included combination of intravenous cloxacillin and ceftriaxone. Patients with severe disease, previously hospitalised in intensive care, or immunocompromised were given clindamycin in addition to previous combination for coverage against MRSA. Cloxacillin combined with gentamicin was given for neonatal sepsis for coverage against both gram positive and negative bacteria. Appropriate surgical intervention was done whenever necessary. Cases of disseminated sepsis were managed according to the guidelines of the institute. Patients were admitted to Intensive care unit as per the requirement $[11,12]$. Specific management included antimicrobial therapy, a thorough surveillance to search for collections and their surgical drainage. Supportive care included maintenance of blood pressure, fluid and electrolyte balance, analgesia, prevention of bed-sores, physiotherapy, asepsis and early enteral nutrition. Patients were followed-up after diagnosis to evaluate the course in the hospital and were followed-up to 3 months after discharge from the hospital.

\section{Results}

\section{Patient Demographics}

During the defined period, 54 patients of disseminated infection were included, of which 25 patients (46.3\%) had osteoarticular infections (14 male and 11 female) with 37 sites of bone/joint involvement seen (lower limb-30 sites and upper limb-7 sites). The predisposing injury was present in 7 (28\%) patients (6-trauma and 1-injection injury). Patients had history of non-significant trauma to the extremities, which correlated with the clinical history and onset of symptoms. The average duration of symptoms was 15.8 days (median 10 days). No known comorbidities of the patients were there before admission.

\section{Clinical Findings}

Fever, swelling and pain in the limb was the presentation at admission in 20 patients and fever and pain was the presentation in three patients. In two patients who had fever alone, the osteoarticular infection was detected after admission within 1 week. Among other organ involvement, pleuro-pulmonary disease was seen in $68 \%$ of patients, soft tissue disease in $64 \%$ patients, venous thrombosis in $24 \%$, and pericardial disease in $16 \%$ patients; 22 out of 25 patients required admission into ICU. Septic arthritis was seen in 17 patients, and osteomyelitis was seen in 12 patients. Multiple sites were involved in eight patients (32\%) with two patients having more than two sites involved, and four patients having both osteomyelitis and septic arthritis. The most common joint was hip (41.6\%) followed by the knee (37.5\%), and the most common bone involved was femur (53.8\%) followed by both humerus and tibia (23.1\% each) (Table 1). The age-wise characteristics have been described in Table 2 . 
Table 1 Characteristics of patients with musculoskeletal infection in disseminated sepsis

\begin{tabular}{llll}
\hline Age distribution & Less than 1 year & 5 \\
\cline { 2 - 3 } & 1-5 years & 6 \\
\cline { 2 - 3 } 6-10 years & 10 \\
\cline { 2 - 3 } Other organ involvement & More than 10 year & 4 \\
& Pleuropulmonary & $68 \%$ \\
& Soft tissue disease & $64 \%$ \\
& Venous thrombosis & $24 \%$ \\
& Pericardial & $16 \%$ \\
Musculoskeletal involvement & Osteomyelitis & Femur & 7 \\
& & Humerus & 3 \\
& & Tibia & 3 \\
& Septic arthritis & Hip & 10 \\
& & Knee & 8 \\
& & Shoulder & 2 \\
& & Ankle & 1 \\
& & Elbow & 1 \\
& & Wrist & 1 \\
\hline
\end{tabular}

\section{Investigations}

The mean total leukocyte count (TLC) among the bone/ joint involved patients was $17,342 / \mathrm{mm}^{3}$, with a median of $15,800 / \mathrm{mm}^{3}$ at admission. C-reactive protein (CRP) was raised among all patients of musculoskeletal involvement with a mean of $138 \mathrm{mg} / \mathrm{L}$ (range 12-365 mg/L; median $=138 \mathrm{mg} / \mathrm{L}$ ). The average erythrocyte sedimentation rate (ESR) value was $46 \mathrm{~mm} / \mathrm{h}$, with a median value of $33 \mathrm{~mm} / \mathrm{h}$. There was no significant difference between musculoskeletal and non-musculoskeletal involved patients of disseminated disease in terms of CRP, ESR and TLC (Table 3). Ultrasonography was suggestive of osteoarticular infection in 20 (80\%) out of 25 patients. Radiography was equivocal in $52 \%$ of patients whereas rest of the $48 \%$ showed signs of infection. MRI was done in four patients and was suggestive of osteoarticular infection in all four of them.

\section{Organisms and Sensitivity}

Fourteen patients had a history of antibiotic usage before admission (four patients-vancomycin, five patients-ceftriaxone, remaining five patients- data could not be retrieved). Out of these 14 patients, positive culture cases were 10, of which eight cases had methicillin-resistant Staphylococcus aureus (MRSA) detected either in their blood or aspirate. In total, out of 25 patients, Staphylococcus aureus (S. aureus) in blood culture was seen in $48 \%$ of patients with MRSA in $28 \%$ and methicillin-sensitive S. aureus (MSSA) in 20\%, and one patient had coagulase-negative Staphylococcus growth. One patient had detection of acinetobacter in repeat blood culture with previous blood culture of MRSA. Joint and bone aspirates showed S. aureus in 56\% with MRSA in 28\% and MSSA in 28\% each and other organisms like E. coli and pseudomonas in $12 \%$. One patient had Pseudomonas stutzeri detected from repeat culture from joint previously detected with E. coli (Table 4). Culture reports comparing musculoskeletal and non-musculoskeletal involved patients shows a relatively higher percentage of $S$. aureus with osteoarticular
Table 2 Age-wise characteristics of patients with osteoarticular infections

\begin{tabular}{|c|c|c|c|c|c|}
\hline Age group & Mean ESR & Mean CRP & Mean TLC & Blood culture & Aspirate culture \\
\hline Neonates $(n=4)$ & 38 & 148 & 16,693 & $\begin{array}{l}\text { MSSA }=1 \\
\text { MRSA }=1 \\
\text { Coag Neg Staph=1 } \\
\text { Sterile }=1\end{array}$ & $\begin{array}{l}\text { MSSA }=1 \\
\text { MRSA }=2 \\
\text { Sterile }=1\end{array}$ \\
\hline 1 month to 1 year $(n=1)$ & 32 & 51 & 12,400 & Sterile & Ps. Aurigenosa \\
\hline 1 to 5 years $(n=6)$ & 51 & 158 & 18,802 & $\begin{array}{l}\text { MRSA }=3 \\
\text { Sterile }=3\end{array}$ & $\begin{array}{l}\text { MSSA }=2 \\
\text { MRSA }=1 \\
\text { E.coli }=1 \\
\text { Sterile }=2\end{array}$ \\
\hline 6 to 10 years $(n=10)$ & 51 & 166 & 15,058 & $\begin{array}{l}\text { MSSA }=4 \\
\text { MRSA }=3 \\
\text { Sterile }=3\end{array}$ & $\begin{array}{l}\text { MSSA }=3 \\
\text { MRSA }=4 \\
\text { Sterile }=3\end{array}$ \\
\hline$>10$ years $(n=4)$ & 37 & 47 & 22,745 & Sterile $=4$ & $\begin{array}{l}\text { MSSA }=1 \\
\text { E.Coli }=1 \\
\text { Sterile }=2\end{array}$ \\
\hline
\end{tabular}

MRSA methicillin-resistant Staphylococcus aureus, MSSA methicillin-sensitive Staphylococcus Aureus, E. coli Escherichia coli, Ps. aurigenosa Pseuomonas aurigenosa, Coag Neg Staph coagulase-negative Staphylococci 
Table 3 Comparison between osteoarticular and nonosteoarticular patients with disseminated infection

\begin{tabular}{lllll}
\hline S. no & Parameter & $\begin{array}{l}\text { Osteoarticular patients } \\
(n=25)\end{array}$ & $\begin{array}{l}\text { Non osteoarticular patients } \\
(n=29)\end{array}$ & $p$ value \\
\hline 1 & Mean ESR & 46 & 42 & 0.26 \\
2 & Mean CRP & 138 & 159 & 0.19 \\
3 & Mean TLC & 17,342 & 16,641 & 0.34 \\
4 & Blood culture & MRSA $=7(28 \%)$ & MRSA $=2(7 \%)$ & NA \\
& MSSA $=7(28 \%)$ & MSSA $=4(14 \%)$ & \\
& E. coli $=2$ & E. coli $=1$ & \\
& Ps. auri $=1$ & Yeast Sp. $=2$ & \\
& Sterile $=8$ & Ps. auri $=1$ & \\
& & S. homi $=1$ & \\
& & Bur Sp $=1$ & \\
& & A. bac $=1$ & \\
& & Kl. Pneu. $=1$ & \\
& & Sterile $=13$ &
\end{tabular}

MRSA methicillin-resistant Staphylococcus aureus, MSSA methicillin sensitive Staphylococcus aureus, E. coli Escherichia coli, Ps. auri Psuedomonas aeruginosa, Kl. pneu Klebshiella pneumoniae, A. bac Acinetobacter, Ps. stut Psuedomonas stutzeri, Bur. Sp Burrelia species, Yeast. Sp yeast species, S. Homi Staphylococcus hominis
Table 4 Culture results in patients with musculoskeletal infection

\begin{tabular}{lll}
\hline Blood culture & MRSA & 7 \\
& MSSA & 7 \\
& E. coli & 2 \\
& Ps. aurigenosa & 1 \\
& Sterile & 8 \\
Aspirate culture & MRSA & 7 \\
& MSSA & 5 \\
& COAG NEG STAPH & 1 \\
Combined blood and & Sterile & Blood culture positive and aspi- \\
aspirate culture results & rate culture negative & 3 \\
& Blood culture negative and aspi- & 7 \\
& rate culture positive & \\
& Both cultures positive & 10 \\
Antibiotic sensitibity & Both cultures negative & 5 \\
among S. aureus & Vancomycin/teicoplanin & $100 \%$ \\
& Clindamycin & $73 \%$ \\
& Oxacillin/methicillin & $46 \%$ \\
\hline
\end{tabular}

MRSA methicillin-resistant Staphylococcus aureus, MSSA methicillin-sensitive Staphylococcus aureus, E. coli Escherichia coli, Ps. aurigenosa Pseuomonas aeruginosa, COAG NEG STAPH coagulasenegative Staphylococci

infections (Table 3). No tuberculosis or fungal cultures were positive.

All $S$. aureus organisms were found sensitive to vancomycin and teicoplanin. Clindamycin sensitivity was seen in S. aureus in $73 \%$, and methicillin/oxacillin sensitivity was seen in $46.2 \%$ cultures. Staph aureus was seen in 15 patients (considering both blood and aspirate). MSSA alone was seen in four patients. MRSA was seen in 11 patients with four out of 11 patients have both MRSA and MSSA cultures (one in blood culture and one in aspirate culture) and have been treated as MRSA positive.

\section{Antibiotic Therapy and Surgery}

Patients received intravenous antibiotics for an average of 3.8 weeks, and the average duration of stay in hospital was 26.9 days (excluding death and discharge against advice). The average duration of intravenous antibiotics given and average duration of stay among the MRSA patients was higher (4.44 weeks and 33.5 days, respectively) compared to MSSA patients (3.33 weeks and 24.2 days) and statistically significant ( $p=0.011$ and $p=0.029$, respectively). IV antibiotics were stopped depending on the improved clinical parameters such as fever, improved joint range of motion, and reduced inflammatory markers like TLC, ESR and CRP. After IV antibiotics, oral antibiotics were given for duration of 3 weeks. Surgery was performed for osteoarticular infections in 21 patients. Repeat surgeries were done in four patients, out of which three patients were positive for MRSA. Two patients were on ventilatory support; one patient was weaned off after 3 days on ventilator and one patient with disseminated staphylococcal disease (MRSA) died after 7 days on ventilator due to sepsis.

The average duration of intravenous antibiotics among the osteoarticular infected patients were higher compared to those without osteoarticular involvement ( 3.8 vs 2.8 weeks, $p=0.017)$ although seven patients in non osteoarticular group and two patients in ostearticular group were not included due to death or left against medical advice. 
CRP values showed a decreasing trend over the course of treatment with a significant difference between MRSA and MSSA patients with a higher initial mean value for MRSA (196 mg/dl) than MSSA (101 mg/dl) $(p=0.024)$. The ESR values over time in MRSA and MSSA and showed higher initial mean value with MRSA (49.7 mm/h) than MSSA $(39.5 \mathrm{~mm} / \mathrm{h})$ but were not statistically significant $(p=0.129)$. Sequalae like ambulatory difficulty, limitation in joint range of movement, limb length discrepancy and deformity were seen in $17.4 \%$ patients ( 3 out of 4 patients were positive for MRSA infection), although the follow-up duration of 3 months is not enough to comment on these parameters (Table 5; Fig. 1).

Table 5 Variation between MRSA and MSSA infected patients

\begin{tabular}{llll}
\hline & MRSA & MSSA & Significance \\
\hline $\begin{array}{l}\text { Mean duration of stay in hospital } \\
\text { (in days) }\end{array}$ & 33.5 & 24.2 & $p<0.05$ \\
$\begin{array}{l}\text { Mean duration of intravenous } \\
\quad \text { antibiotics (in weeks) }\end{array}$ & 4.44 & 3.33 & $p<0.05$ \\
$\begin{array}{l}\text { Mean initial CRP (mg/dl) } \\
\text { Mean initial ESR (in mm/h) }\end{array}$ & 196 & 101 & $p<0.05$ \\
\hline
\end{tabular}

\section{Discussion}

Despite many studies of septic arthritis and osteomyelitis in children, there is a paucity of studies regarding the prevalence of such infections in cases of disseminated disease. In our study, the prevalence of osteoarticular infections among the patients of disseminated disease was around $43 \%$, with a predominance of lower extremity involvement. Septic arthritis was seen in $27.8 \%$ of patients, and osteomyelitis was seen in $22.2 \%$ of patients of disseminated infection. $7.4 \%$ of patients had both concurrent septic arthritis and adjacent osteomyelitis. Miles reviewed the admission of disseminated staphylococcal infections in PICU, among which osteoarticular infections were higher with septic arthritis and osteomyelitis in $39.7 \%$ and $55.2 \%$, respectively [13]. This may be attributed to a major number of patients $(79 \%)$ having musculoskeletal symptoms on presentation.

Among the osteoarticular infected patients, pleuropulmonary disease was seen in $68 \%$ of patients in our study. Mortality was seen in 13\% (7 patients) among all the cases of disseminated infection with one patient of osteoarticular involvement. Paterson noted in his study that pneumonia and empyema were detected in $87 \%$ and $53 \%$, respectively, among the 38 patients with osteoarticular infection with disseminated staphylococcal disease. The mortality among these patients was $13 \%$ [14].


Fig. 1 Trends in ESR and CRP values among MRSA and MSSA infected patients showing significant difference $(p<0.05)($ MRSA-blue line; MSSA-green line) 
Predisposing history of injury was seen in $28 \%$ among the 25 cases of osteoarticular involvement. Miles reported a higher number in his study, in which $67 \%$ of patients had disseminated sepsis, and $53.4 \%$ had a history of minor trauma to limb in the preceding month [15]. The significance of this history is doubtful, but it could be a potential index site from which infection has disseminated or has caused osteoarticular infection followed by its bacteremia and dissemination.

The hip (43.5\%) and knee (34.8\%) were the most common joints involved. With respect to osteomyelitis, the femur was most commonly involved (46.1\%). Previous studies also ascertained this finding with the hip found to be the most common site among septic arthritis and femur found to be the most common site among osteomyelitis [15]. Paterson found out that hip was commonly involved among septic arthritis (55.5\%), and the involvement of tibia (48.8\%) and femur $(41.4 \%)$ was higher in osteomyelitis among the patients of disseminated staphylococcal disease in his study [10]. Four out of the 25 patients (16\%) had involvement of septic arthritis with adjacent bone osteomyelitis in our study. Perlman et al. found a higher incidence (33\%) of similar bone and joint involvement in his study [16].

Ultrasound was positive for effusion or collection in $80 \%$ among all 25 osteoarticular infected patients. Among septic arthritis patients alone, $94.1 \%$ of patients had positive findings in USG. Ultrasonography for symptomatic hip effusions has sensitivity of $85-93.4 \%$ and a specificity of $93-100 \%$ [17, 18]. Ultrasound is more useful in suspected septic arthritis although periosteal abnormalities may be visualized in osteomyelitis, but its disadvantage is its user dependency [19]. On the other hand, bone scan and MRI are useful investigations for osteomyelitis. ${ }^{99 \mathrm{~m}} \mathrm{Tc}-$ MDP is a highly sensitive test but has less specificity [20, 21]. A bone scan can be useful when the multifocal disease is suspected and hence can be useful in cases of disseminated disease. However, the test is non-specific, may require further clinical and radiological correlation with MRI or radiograph, and also is not widely available in our country. A high index of suspicion must be maintained for evaluation of children who present with clinical signs and symptoms worrisome for musculoskeletal infection. Unexplained limp, reluctance to use a limb or inability to weight bear, bone or joint pain with a hot and swollen joint, bone or joint tenderness and complete reluctance to move a joint or limb suggest further evaluation. If any neonate had irritability or poor feeding, then careful examination is necessary. Any swelling, erythema, decreased range of motion, lack of movement of involved limb, or pain on movement should raise our suspicion for further investigations. Plain radiographs and laboratory studies (CBC with differential, CRP, ESR, and blood cultures) are sent for, ultrasonography is performed for collection and aspirates are sent for microbiological analysis (gram stain and aerobic bacterial culture, TB culture and fungal culture and sensitivity). When uncertainty persists despite a thorough evaluation in a child with worrisome musculoskeletal complaints, close clinical follow-up is necessary until either the problem resolves or further evidence leads to a specific diagnosis. A supplemental study with bone scintigraphy or magnetic resonance imaging (MRI) may be done to further clarify the diagnosis and guide the treatment process. The presence of large collections, thick pus, joint locations, and pus evacuating into surrounding soft tissues generally require surgical drainage.

The values of ESR and CRP were raised in $100 \%$ and $92 \%$, respectively. Bonhoeffer reviewed 90 patients of osteoarticular infections in which ESR and CRP found to be raised in $100 \%$ and $82 \%$, respectively. These values of ESR and CRP were also raised in other patients of disseminated disease in our study since they are inflammatory markers, hence being more useful as a prognostic indicator for therapy rather than for the diagnosis of osteoarticular infections in such cases of disseminated disease [22].

Blood culture results were positive in $52 \%$ of patients. Among all the patients with positive blood culture, growth of $S$. aureus with methicillin-resistant strains accounted for around half. Joint and bone aspirate cultures showed higher results with $68 \%$ positive cultures, the majority of which were $S$. aureus with equally distributed number among methicillin-sensitive and methicillin-resistant strains. Overall, $64 \%$ of patients had S. aureus detected in either blood or tissue culture. Bonhoeffer reviewed the patients of osteoarticular infections and found similar results [22]. In his study, blood and tissue cultures were positive in $52 \%$ and $57 \%$ cultures, respectively.

In cases where both blood culture and tissue culture was performed, $35 \%$ had both cultures positive, and 23\% had both cultures negative. Standard culture methods may fail to show positive cultures in many cases. Culture negative results may be due to less virulent organisms, due to slowgrowing organisms, due to the low burden of disease, or due to the administering of antibiotics (Table 2).

Staphylococcus aureus is found commonly in all age groups upto 12 years. Streptococcus species, Enterobacteriaceae and Escherichia coli are seen in neonates. Kingella kingae, Streptococcus pneumoniae, Neisseria meningitidis, and Haemophilus influenzae type b are seen in both infantile and early childhood groups (upto 3 years). Group A betahemolytic streptococcus is seen in children (3-12 years) [23]. Overall, 32 patients of disseminated sepsis had $S$. aureus detected in either blood or aspirate, out of which MRSA was found in 20 patients, and MSSA was found in 12 patients. Among these, 55\% of MRSA infected patients and $50 \%$ of MSSA infected patients were found to have osteoarticular involvement. Hence the need for careful search of osteoarticular infections in all cases of disseminated disease, 
with more emphasis in cases of disseminated staphylococcal disease, is imminent.

Surgery was performed for osteoarticular infections in 21 patients. 1 patient left against medical advise and remaining three patients that did not underwent surgery were admitted for average of 3 weeks. These patients did not have abscess or collection and have responded and improved with intravenous antibiotics alone. In acute and sub-acute osteomyelitis, surgery is not typically required unless the clinical condition has not improved in response to antibiotic therapy [23]. Clinical response and CRP levels may help to monitor the ongoing treatment and need for further surgical interventions. Four patients required repeated surgeries either due to reaccumulation in previous sites (two patients) or due to the development of new sites of infection (two patients). Of these four patients, one had MSSA detected in aspirate, and three patients had MRSA detected in blood/aspirate culture. Paterson has reviewed 38 patients of osteoarticular infections with disseminated staphylococcal disease, in which 12 patients required repeated surgery due to similar reasons [14]. There may be a higher chance of repeat surgery as disseminated disease possibly may cause a state of immunocompromise and facilitate in the development of new sites or reaccumulation in old sites. MRSA strain itself may be another factor in such a scenario as it is considered more virulent than MSSA [24]. Also, it is likely that metastatic foci might get settled during the early bacteremia in the case of disseminated disease hence leading to the development of new sites of infection [25].

Methicillin-resistant Staphylococcus aureus (MRSA) has been increasingly reported in recent years. In the United States, MRSA has been detected in around one-third of osteoarticular infections [26]. In a recent study in a tertiary care center in India, MRSA was the predominant isolate in $13 \%$ of young infants with septic arthritis [27]. In our study, patients of MRSA (excluding death and left against medical advice) have received a longer duration of intravenous antibiotics and longer duration of stay in hospital compared to MSSA patients. Also, three out of the four patients with repeated surgeries had MRSA infection. MRSA could be a concern due to its increased virulence, probably due to panton leukocidin toxin. It has been found to be associated with a severe osteoarticular disease, including extended hospital stays, the need for longer antibiotic administration, and more surgical interventions [24]. Furthermore, on the analysis of serial CRP values, there was a statistical difference in the overall values between the MRSA infected patients and MSSA infected patients of osteoarticular involvement in our study and pave the way for further large studies to detect such difference.

Two patients had reported the presence of mixed flora, one via blood culture and one via joint aspirate culture. The reasons for the occurrence of mixed flora or mixed infection in musculoskeletal infections is not exactly known but has been reported in the literature. Mixed infection may cause a delayed clinical response after antibiotic treatment and joint debridement [28].

Osteoarticular infections are high among cases of disseminated disease, and increased clinical suspicion for such must be maintained. Screening of these infections by frequent examination and comprehensive imaging, if and when required, can go a long way in improving the outcome of the patients. Ultrasonography is a useful investigation with higher sensitivity for suspected septic arthritis. MRSA is a cause of severe osteoarticular infections with increased morbidity, and the need for proper empirical treatment is imminent in cases of disseminated disease. Vancomycin, along with aminoglycoside, can be the preferred choice of antibiotic in cases of disseminated disease with suspected osteoarticular infection, as all cases of Staphylococcus aureus were sensitive to vancomycin in our study, but further studies are requires to ascertain its use before recommending it as a first choice drug [29]. PCR may be useful for differentiating MRSA from MSSA in a short time, but cost factors and universal availability can hinder its usage. Future research should raise awareness of musculoskeletal involvement in such cases of disseminated sepsis and could focus on further prospective assessments of proper antibiotic treatment regimens and diagnostic protocols, such as PCR, that may allow earlier diagnosis of osteoarticular infections to reduce long term morbidity. Also, new and bigger studies can be of paramount importance to further evaluate MRSA versus MSSA in osteoarticular infections. Limited followup and small sample size were the limitations of this study.

\section{Conclusion}

The prevalence of osteoarticular infections is high among pediatric patients of disseminated disease and increased clinical suspicion for such must be maintained. Hip and knee are the most common sites for septic arthritis, while femur is the most common site for osteomyelitis in patients with disseminated disease, as per the study. Cases of osteoarticular involvement with MRSA were higher compared to MSSA among the cases of disseminated disease with serial CRP values in MRSA patients were higher and statistically significant compared to MSSA patients. MRSA patients with osteoarticular involvement may require a longer duration of intravenous antibiotics, longer duration of hospital stay, and may have less satisfactory outcomes when compared to MSSA.

Funding None. 


\section{Declarations}

Conflict of Interest The authors declare that they have no conflict of interest.

Ethical Approval The study was approved by Institute Ethics Committee vide number INT/IEC/2017/000310.

Informed Consent Informed consent had been obtained from the parents of the children included in the study.

\section{References}

1. Sukswai, P., Kovitvanitcha, D., Thumkunanon, V., Chotpitayasunondh, T., Sangtawesin, V., \& Jeerathanyasakun, Y. (2011). Acute hematogenous osteomyelitis and septic arthritis in children: clinical characteristics and outcomes study. Journal of Medical Association of Thailand, 94, S209-S216.

2. Akinkugbe, O., Stewart, C., \& McKenna, C. (2019). Presentation and investigation of pediatric bone and joint infections in the pediatric emergency department. Pediatric Emergency Care, 35, 700-704. https://doi.org/10.1097/PEC.0000000000001431

3. Iliadis, A. D., \& Ramachandran, M. (2017). Paediatric bone and joint infection. EFORT Open Reviews, 2, 7-12. https://doi.org/10. 1302/2058-5241.2.160027

4. Borella, L., Goobar, J. E., Summitt, R. L., \& Clark, G. M. (1963). Septic arthritis in childhood. Journal of Pediatrics, 62, 742-747. https://doi.org/10.1016/s0022-3476(63)80044-x

5. McCarthy, J. J., Dormans, J. P., Kozin, S. H., \& Pizzutillo, P. D. (2005). Musculoskeletal infections in children: basic treatment principles and recent advancements. Instructional Course Lectures, 54, 515-528.

6. Watson, R. S., Carcillo, J. A., Linde-Zwirble, W. T., Clermont, G., Lidicker, J., \& Angus, D. C. (2003). The epidemiology of severe sepsis in children in the United States. American Journal of Respiratory and Critical Care Medicine, 167, 695-701.

7. Mandal, K., Roy, A., Sen, S., Bag, T., Kumar, N., \& Moitra, S. (2014). Disseminated staphylococcal disease in healthy childrenexperience from two tertiary care hospitals of West Bengal. Indian Journal of Pediatrics, 81, 133-137. https://doi.org/10.1007/ s12098-013-1034-7

8. Verma, R., Patil, T. B., \& Lalla, R. (2012). Disseminated tuberculosis manifesting as pulmonary, meningeal and spinal tuberculosis in an immunocompetent patient. BMJ Case Reports, 2012, bcr2012007778. https://doi.org/10.1136/bcr-2012-007778

9. Morrey, B. F., Bianco, A. J., Jr., \& Rhodes, K. H. (1975). Septic arthritis in children. Orthopedic Clinics of North America, 6 , 923-934.

10. Peltola, H., \& Vahvanen, V. (1984). A comparative study of osteomyelitis and purulent arthritis with special reference to aetiology and recovery. Infection, 12(2), 75-79. https://doi.org/10.1007/BF016 41675

11. Singhi, S. (1994). Intensive care of pediatric patient. In H. P. S. Sachdev, R. K. Puri, A. Bagga, \& P. Choudhury (Eds.), Principles of pediatric and neonatal intensive care (pp. 329-353). Jaypee Brothers.

12. Baranwal, A. K., Singhi, S. C., \& Jayashree, M. (2007). A 5-year PICU experience of disseminated staphylococcal disease, part 1: clinical and microbial profile. Journal of Tropical Pediatrics, 53(4), 245-251. https://doi.org/10.1093/tropej/fmm022

13. Miles, F., Voss, L., Segedin, E., \& Anderson, B. J. (2005). Review of Staphylococcus aureus infections requiring admission to a paediatric intensive care unit. Archives of Disease in Childhood, 90, $1274-1278$
14. Paterson, M. P., \& Hoffman, E. B. (1990). Severe disseminated staphylococcal disease associated with osteitis and septic arthritis. Journal of Bone and Joint Surgery. British Volume, 72, 94-97.

15. Maraqa, N. F., Gomez, M. M., \& Rathore, M. H. (2002). Outpatient parenteral antimicrobial therapy in osteoarticular infections in children. Journal of Pediatric Orthopedics, 22, 506-510.

16. Perlman, M. H., Patzakis, M. J., Kumar, P. J., \& Holtom, P. (2000). The incidence of joint involvement with adjacent osteomyelitis in paediatric patients. Journal of Pediatric Orthopedics, 20, 40-43.

17. Vieira, R. L., \& Levy, J. A. (2010). Bedside ultrasonography to identify hip effusions in pediatric patients. Annals of Emergency Medicine, 55(3), 284-289. https://doi.org/10.1016/j.annemergmed. 2009.06.527 Epub 2009 Aug 20 PMID: 19695738.

18. Mnif, J., Khannous, M., Keskes, H., Louati, N., Damak, J., \& Kechaou, M. S. (1997). L'échographie dans l'approache diagnostique des arthrites septiques [Ultrasonography in the diagnostic approach of septic arthritis]. Revue de Chirurgie Orthopedique et Reparatrice de l'Appareil Moteur, 83(2), 148-155. French. PMID: 9231182.

19. Dartnell, J., Ramachandran, M., \& Katchburian, M. (2012). Haematogenous acute and subacute paediatric osteomyelitis: a systematic review of the literature. Journal of Bone and Joint Surgery. British Volume, 94, 584-595.

20. Littenberg, B., \& Mushlin, A. I. (1992). Technetium bone scanning in the diagnosis of osteomyelitis: a meta-analysis of test performance. Journal of General Internal Medicine, 7, 158-163.

21. Kocher, M. S., Lee, B., Dolan, M., Weinberg, J., \& Shulman, S. T. (2006). Pediatric orthopedic infections; early detection and treatment. Pediatric Annals, 35, 112-122.

22. Bonhoeffer, J., Haeberle, B., Schaad, U. B., \& Heininger, U. (2001). Diagnosis of acute haematogenous osteomyelitis and septic arthritis: 20 years experience at the University Children's Hospital Basel. Swiss Medical Weekly, 131, 575-581.

23. Dodwell, E. R. (2013). Osteomyelitis and septic arthritis in children: current concepts. Current Opinion in Pediatrics, 25, 58-63. https:// doi.org/10.1097/MOP.0b013e32835c2b42

24. Otto, M. (2012). MRSA virulence and spread. Cellular Microbiology, 14, 1513-1521.

25. Lesens, O., Hansmann, Y., Brannigan, E., Remy, V., Hopkins, S., Martinot, M., Meyer, P., Connel, B. O., Monteil, H., Christmann, D., $\&$ Bergin, C. (2004). Positive surveillance blood culture is a predictive factor for secondary metastatic infection in patients with Staphylococcus aureus bacteraemia. Journal of Infection, 48, 245-252.

26. Arnold, S. R., Elias, D., Buckingham, S. C., Thomas, E. D., Novais, E., Arkader, A., \& Howard, C. (2006). Changing patterns of acute hematogenous osteomyelitis and septic arthritis: emergence of community associated methicillin-resistant Staphylococcus aureus. Journal of Pediatric Orthopedics, 26, 703-708.

27. Sankaran, G., Zacharia, B., Roy, A., \& Purayil, S. P. (2018). Current clinical and bacteriological profile of septic arthritis in young infants: a prospective study from a tertiary referral centre. European Journal of Orthopaedic Surgery and Traumatology, 28, 573-578.

28. Umer, M., Hashmi, P., Ahmad, T., Ahmed, M., \& Umar, M. (2003). Septic arthritis of the hip in children-Aga Khan University Hospital experience in Pakistan. The Journal of the Pakistan Medical Association, 53, 472-478.

29. Kabak, S., Halici, M., Akcakus, N. C., \& Narin, N. (2002). Septic arthritis in patients followed-up in neonatal intensive care unit. Pediatrics International, 44, 652-657. https://doi.org/10.1046/j.1442200x.2002.01649.x

Publisher's Note Springer Nature remains neutral with regard to jurisdictional claims in published maps and institutional affiliations. 